\title{
Size Effect of Encaged Clusters on the Exohedral Chemistry of Endohedral Fullerenes: A Case Study on the Pyrrolidino Reaction of $\mathrm{Sc}_{\mathrm{x}} \mathrm{Gd}_{3-\mathrm{x}} \mathbf{N} @ \mathrm{C}_{80}(\mathrm{x}=\mathbf{0 - 3})$ \\ Ning Chen ${ }^{\mathrm{a}, \mathrm{b}}$, Er-Yun Zhang ${ }^{\mathrm{a}, \mathrm{b}}$, Kai Tan, ${ }^{\mathrm{c}}$ Chun-Ru Wang ${ }^{\mathrm{a},{ }^{*}}$ Xin Lu ${ }^{\mathrm{c},{ }^{*}}$ \\ ${ }^{a}$ Beijing National Laboratory for Molecular Sciences (BNLMS), Institute of Chemistry, Chinese Academy of Sciences (CAS), Beijing 100080, China. ${ }^{b}$ Graduate University of CAS, Beijing, China. 'State Key Laboratory of Physical Chemistry of Solid Surfaces \& Center for Theoretical Chemistry, College of Chemistry and Chemical Engineering, Xiamen University, Xiamen, 361005, China.
}

S1. The synthesis and HPLC separation of $\mathrm{Sc}_{\mathrm{x}} \mathrm{Gd}_{3-\mathrm{x}} \mathrm{N} @ \mathrm{C}_{80}(\mathrm{x}=\mathbf{0 - 3})$

Mixture of Sc/Ni alloy, Gd/Ni alloy and graphite powder were pressed into the holes of graphite-rod electrodes in a molar Sc:Gd:C ratio of 1:1:24. As the resource of nitrogen, 3Torr $\mathrm{N}_{2}$ was introduced to reactor at the atmosphere of 300 Torr He. The fullerene soot generated was Soxlet-extracted with toluene and purified by HPLC. Multi-stage HPLC technique is employed to isolate and purify the endohedral fullerenes, using two complementary columns: Cosmosil Buckyprep, and Cosmosil Buckyprep-M.

$\mathrm{Sc}_{3} \mathrm{~N} @ \mathrm{C}_{80}$ were isolated from the soot extract ( $\mathrm{Sc} / \mathrm{C}$ composite rods in arc-discharge) and purified via a two-stage of high-performance liquid chromatography (HPLC). Buckyprep column separates fullerenes according to their size. In the first stage, the Buckyprep column was used to collect the $\mathrm{C}_{88}-\mathrm{C}_{90}$ fraction. Buckyprep-M separates fullerenes according to their polarity (similar to Buckyclutcher column), which was employed in the second stage to remove the hollow fullerenes $\left(\mathrm{C}_{88}, \mathrm{C}_{90}\right)$ from $\mathrm{Sc}_{3} \mathrm{~N} @ \mathrm{C}_{80}$. The stage 1 and 2 were repeated several times until pure $\mathrm{Sc}_{3} \mathrm{~N} @ \mathrm{C}_{80}$ being collected. $\mathrm{Gd}_{3} \mathrm{~N} @ \mathrm{C}_{80}$ was separated via a similar process (soot extract from $\mathrm{Gd} / \mathrm{C}$ composite rods arc-discharge) as $\mathrm{Sc}_{3} \mathrm{~N} @ \mathrm{C}_{80}$, only the collection fractions are different for them due to the different retention times of $\mathrm{Sc}_{3} \mathrm{~N} @ \mathrm{C}_{80}$ and $\mathrm{Gd}_{3} \mathrm{~N} @ \mathrm{C}_{80}$.

$\mathrm{Sc}_{2} \mathrm{GdN} @ \mathrm{C}_{80}$ and $\mathrm{ScGd}_{2} \mathrm{~N} @ \mathrm{C}_{80}$ were isolated from the soot extract $(\mathrm{Sc} / \mathrm{Gd} / \mathrm{C}$ composite rods arc-discharge) and purified via a four-stage HPLC process. The first stage used the Buckyprep column to collect the $\mathrm{C}_{88}-\mathrm{C}_{90}$ fraction, the second stage is performed in a Buckyprep-M column to remove the hollow fullerenes. The third stage of HPLC utilized Buckyprep column again but recycling function is employed to separate $\mathrm{Sc}_{3} \mathrm{~N} @ \mathrm{C}_{80}, \mathrm{Gd}_{3} \mathrm{~N} @ \mathrm{C}_{80}$, 
$\mathrm{Sc}_{2} \mathrm{GdN} @ \mathrm{C}_{80}, \mathrm{ScGd}_{2} \mathrm{~N} @ \mathrm{C}_{80}$ into four different fractions. The fourth stage HPLC used the Buckyprep column in recycling mode to purify the isolated $\mathrm{Sc}_{2} \mathrm{GdN} @ \mathrm{C}_{80}$ and $\mathrm{ScGd}_{2} \mathrm{~N} @ \mathrm{C}_{80}$.

a)

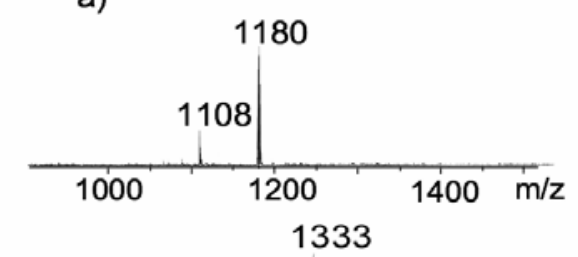

c)

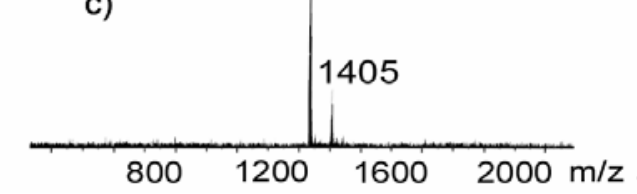

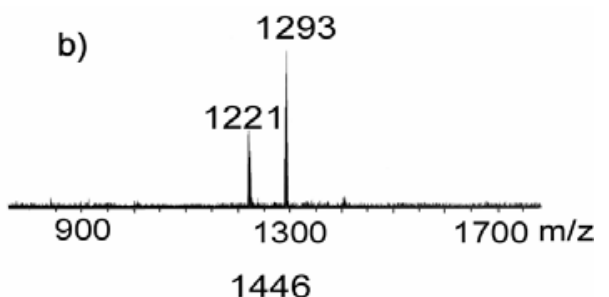

d)

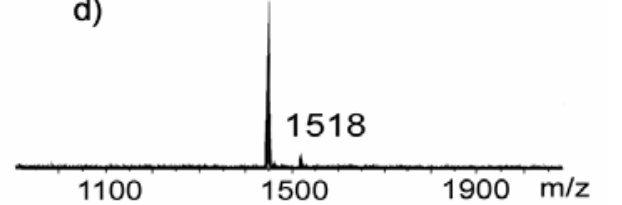

Figure S1. MALDI-MS of the monoadducts of pyrrolidinofullerenes. a) $\mathrm{Sc}_{3} \mathrm{~N} @ \mathrm{C}_{80}$, b) $\mathrm{Sc}_{2} \mathrm{GdN} @ \mathrm{C}_{80}$, c) ScGd $\mathrm{Sc}_{2} \mathrm{~N} @ \mathrm{C}_{80}$ and d) $\mathrm{Gd}_{3} \mathrm{~N} @ \mathrm{C}_{80} . \mathrm{Sc}_{3} \mathrm{~N} @ \mathrm{C}_{80}, \mathrm{Sc}_{2} \mathrm{GdN} @ \mathrm{C}_{80}, \mathrm{ScGd}_{2} \mathrm{~N} @ \mathrm{C}_{80}$ and $\mathrm{Gd}_{3} \mathrm{~N} @ \mathrm{C}_{80}$ are fragments of the parent pyrrolidinofullerenes under laser desorption.

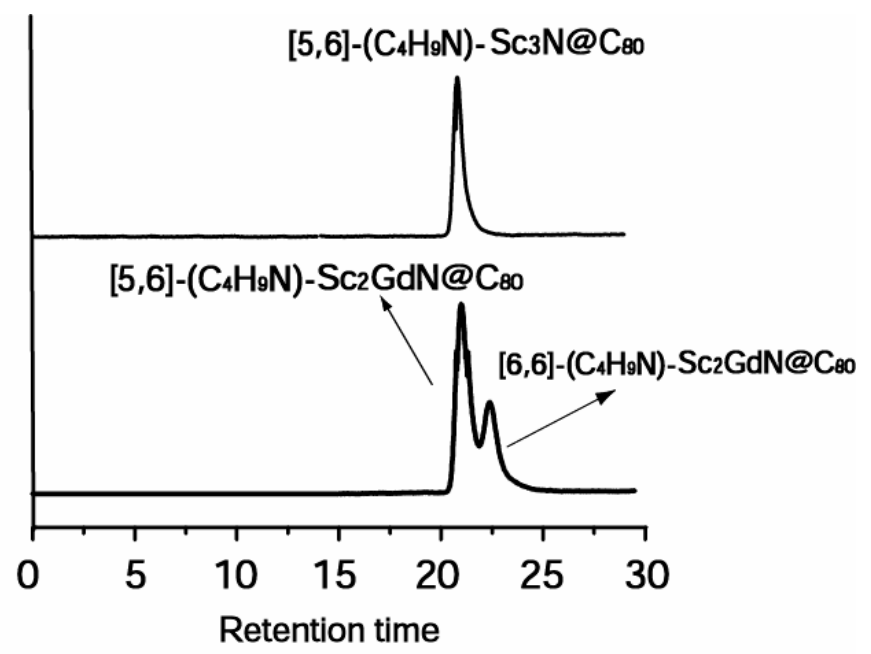

Figure S2. Comparative study of the HPLC (Buckyprep-M, toluene, $12 \mathrm{ml} / \mathrm{min}$ ) profiles of $[5,6]-$ pyrrolidino- $\mathrm{Sc}_{3} \mathrm{~N} @ \mathrm{C}_{80}$ and [5,6]/[6,6]-pyrrolidino- Sc $2 \mathrm{GdN} @ \mathrm{C}_{80}$ 
Endofullerenes

Pyrrolidinofullerenes

$\mathrm{Sc}_{x} \mathbf{G d}(3-x) \mathbf{N} @ \mathrm{C}_{80}$ $(x=0-3)$
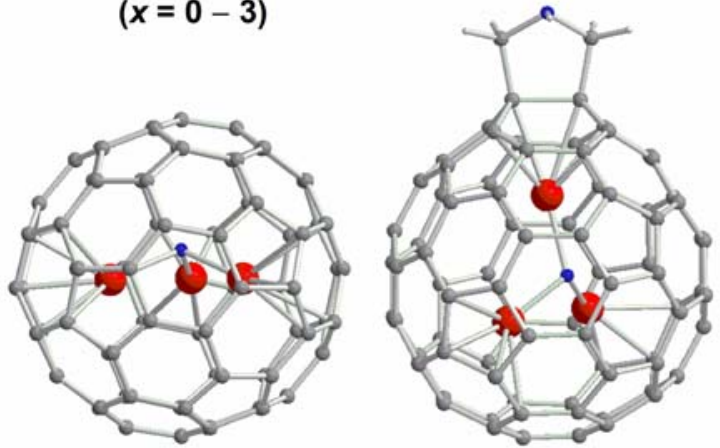

$[5,6]$-adduct

$\mathrm{Gd}_{3} \mathrm{~N} @ \mathrm{C}_{80}$
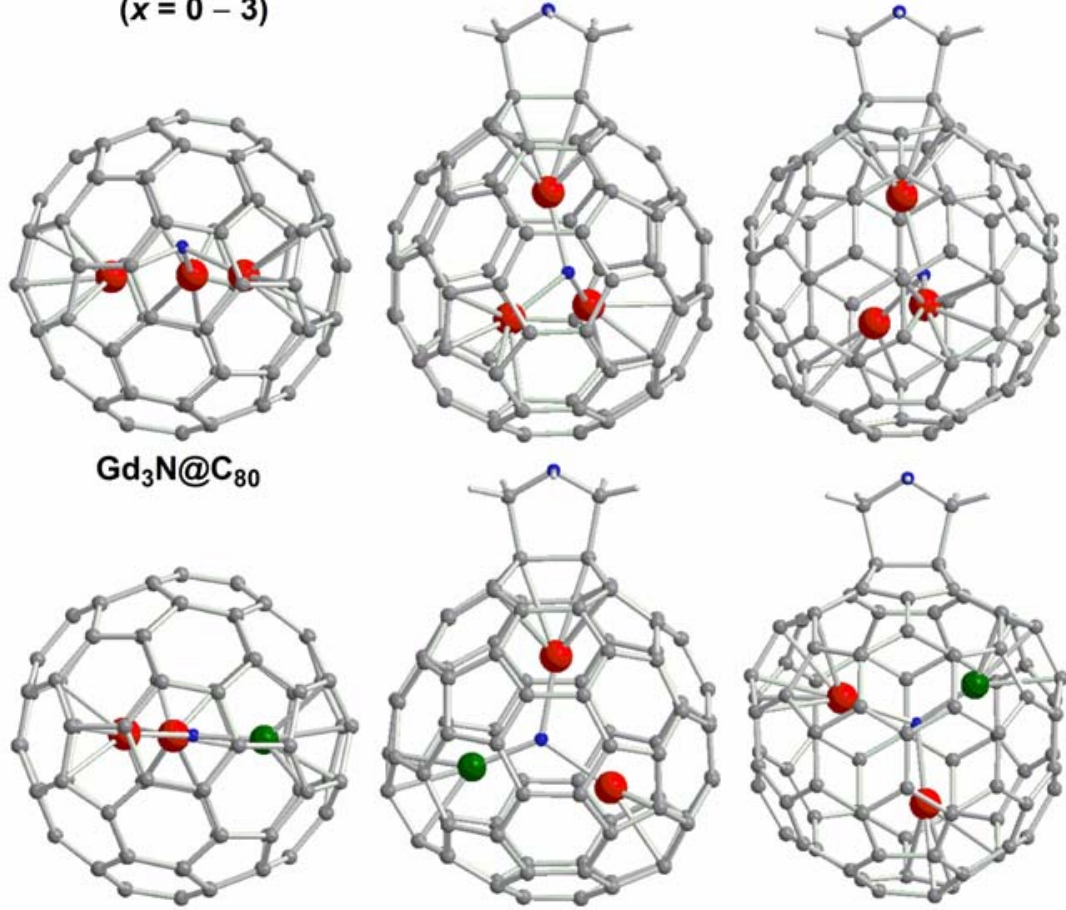

$\mathrm{ScGd}_{2} \mathrm{~N} @ \mathrm{C}_{80}$
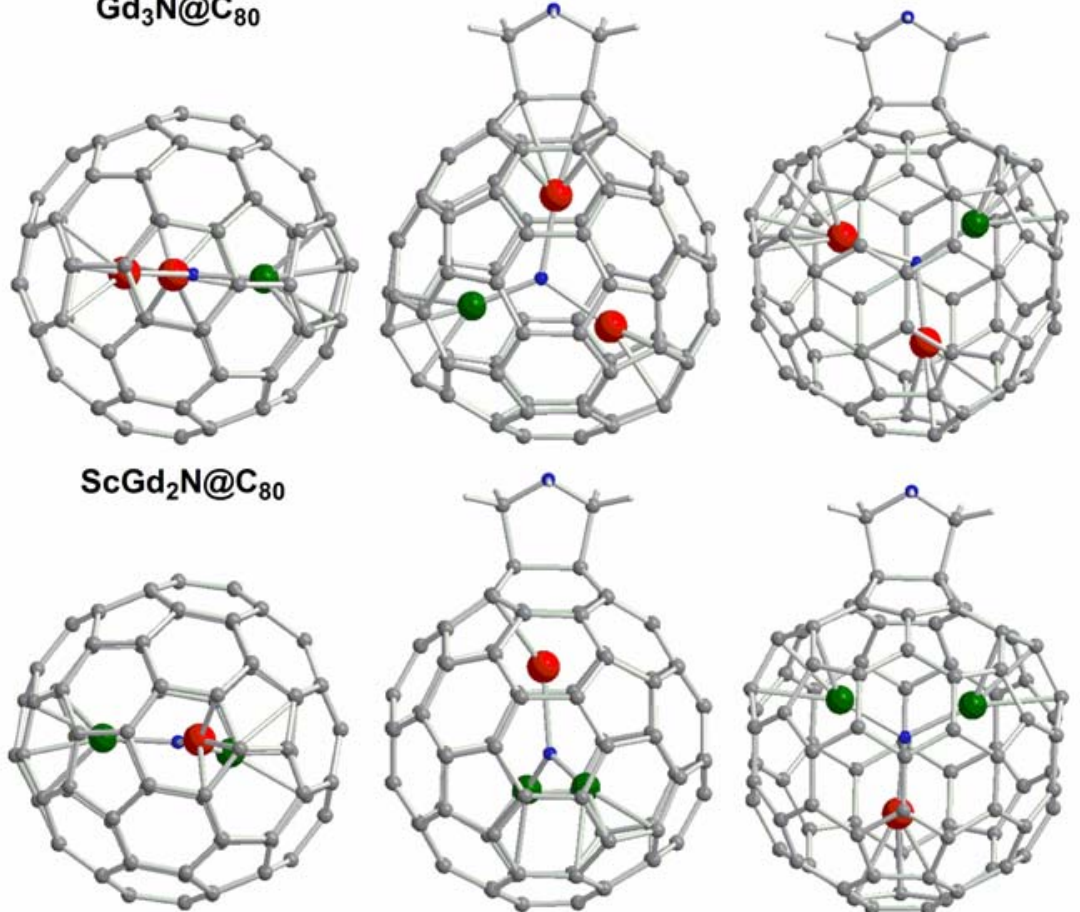

$\mathrm{Sc}_{2} \mathrm{GdN} @ \mathrm{C}_{80}$
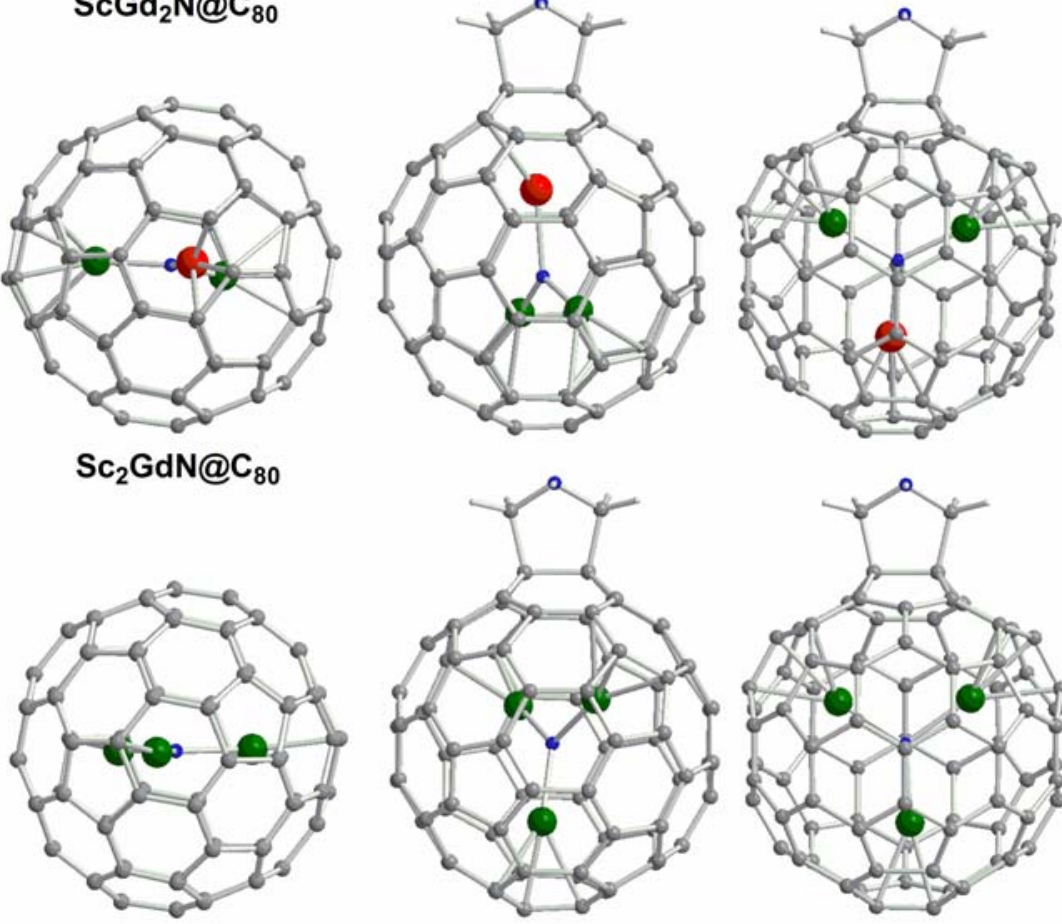

$\mathrm{Sc}_{3} \mathrm{~N} @ \mathrm{C}_{80}$

Figure S3 PBE/DNP-predicted geometries of $\mathrm{Sc}_{\mathrm{x}} \mathrm{Gd}_{3-\mathrm{x}} \mathrm{N} @ \mathrm{C}_{80}(\mathrm{x}=0-3)$ and the [6,6]- and [5,6]-regioisomers of their pyrrolidino-adducts. 